\title{
New Brunswick
}

\section{Anaerobic yeast fermentation for the production of ethanol in a versatile lab fermentor}

\author{
Whether used for research or production, the versatile BioFlo ${ }^{\oplus} 310$ fermentor from New Brunswick \\ Scientific allows growth of a wide variety of aerobic and anaerobic microorganisms, including bacteria, \\ plant, algae, fungi and yeast. Its advanced controller can regulate up to four vessels simultaneously, 120 \\ process loops in all. Here we demonstrate one facet of its versatility - a technique for inducing ethanol \\ production in yeast, by switching from an aerobic growth phase to an anaerobic steady-state culture.
}

Saccharomyces cerevisiae is a model eukaryotic organism, often used in research because it is easy to manipulate and culture, and is comparatively similar in structure to human cells. This yeast is also widely used in industrial applications to manufacture enzymes and proteins for beer, wine and bread, and because it metabolizes glucose to ethanol, is also used to produce many biofuel products. We produced ethanol from a S. cerevisiae (American Type Culture Collection strain 20602) in a 7.5 liter BioFlo 310 fermentor, to demonstrate the flexibility of this advanced fermentation system. In the first phase, we grew the yeast in an aerobic environment, using a dissolved oxygen cascade control strategy to produce a sufficient cell density. Then we pumped in nitrogen gas to create an anaerobic environment for inducing ethanol production, and used reduction and oxidation (redox) potential measurements to monitor any increase in dissolved oxygen levels, which signaled a slowdown in cell growth. Redox potential activates ethanol production and changes the total soluble protein pattern of $S$. cerevisiae ${ }^{1}$. We used redox potentials to control the oxidation-reduction level by adding feed medium to the vessel when the redox potential value in the fermentor vessel rose above-130 mV.

\section{Advanced control in a compact package}

The BioFlo 310 fermentor (Fig. 1) consists of a master control station with built-in controller, 15-inch color touchscreen display, three built-in pumps and 4-gas mixing with a thermal mass flow controller for gas flow control. The BioFlo 310 is available with choice of four interchangeable autoclavable vessels, 2.5, 5.0, 7.5 and 14.0 liter total volume, and includes a $\mathrm{pH}$ probe, dissolved oxygen probe and level or foam probe, as well as hoses, sterile sampler and more. The system can

\section{Yinliang Chen, Jeff Krol, Weimin Huang, Rich Mirro \& Vik Gossain}

R\&D Laboratory, New Brunswick Scientific, 44 Talmadge Road, Edison, New Jersey 08818 , USA. Correspondence should be addressed to V.G. (vik.gossain@nbsc.com). be operated in batch, fed-batch and continuous modes for handling a variety of applications, and it meets current good manufacturing practice (cGMP) requirements.

Here we used a 7.5-liter vessel and operated the system in fed-batch mode. We added a Mettler-Toledo redox sensor to the vessel and directly connected it to the fermentor controller's optional redox input. We used New Brunswick Scientific's optional BioCommand ${ }^{\circledR}$ Plus with OPC Control software to automatically control the process and log data throughout the run. Ethanol production and glucose concentrations were measured off-line by YSI's 2700 Select $^{\mathrm{TM}}$, Biochemistry Analyzer, and we used a Beckman-Coulter Vi-Cell ${ }^{\mathrm{TM}}$ XR Cell Viability Analyzer to measure the cell viability and concentration during the entire process.

Inoculum preparation and growth phase

We prepared a seed culture in a one-liter Erlenmeyer flask containing $250 \mathrm{ml}$ of DIFCO YM growth medium (Becton Dickinson), using a

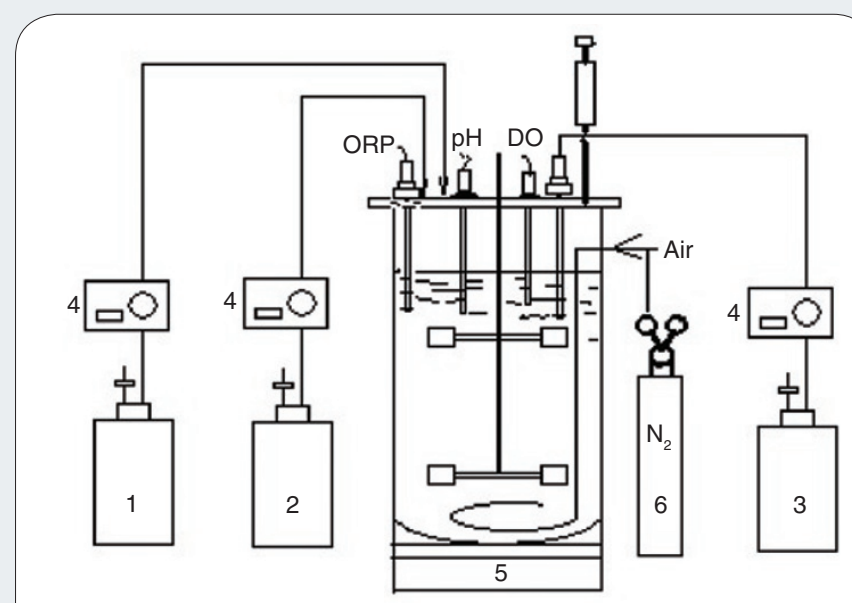

Figure 1 | BioFlo310 setup for anaerobic growth. Shown are containers for base solution (1) and feed medium (2), a collection reservoir (3), three pumps (4), the fermentor vessel with $\mathrm{pH}$, dissolved oxygen (DO) and redox (OPR) probes (5), and a nitrogen gas tank (6). 


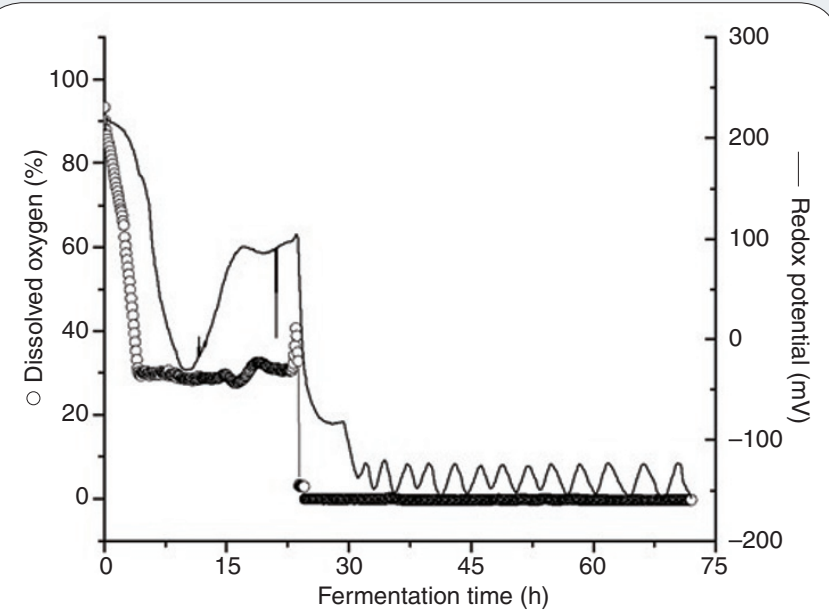

Figure $\mathbf{2}$ |Time profiles of dissolved oxygen and redox measurements during $\mathrm{S}$ cerevisiae fermentation. Dissolved oxygen was controlled at $30 \%$ during the cell growth phase followed by an anaerobic production phase in nitrogen gas.

$1.0 \mathrm{ml}$ frozen suspension. The culture was incubated at $29^{\circ} \mathrm{C}$ for 18 hours at 240 r.p.m. in an orbital shaker (New Brunswick Scientific model Innova ${ }^{\circledR} 43 R$ ). Then we transferred the inoculum to the BioFlo 310 vessel containing 4.75 liters of fermentation medium. The fermentor was controlled at $30^{\circ} \mathrm{C}$ and $\mathrm{pH}$ 5.0. Aeration rate was set at $2.5 \mathrm{I}$ $\min ^{-1}$ (0.5 vessel volumes per minute), and agitation speed was 200800 r.p.m. We used a $29 \% \mathrm{NH}_{4} \mathrm{OH}$ base solution to control $\mathrm{pH}$ and $50 \%$ glucose as feed medium. Dissolved oxygen and redox potential were measured during the entire process (Fig. 2).

Dissolved oxygen was cascade-controlled at $30 \%$ via agitation. The optical density of the fermentation broth was measured at $600 \mathrm{~nm}$ to monitor cell growth. Glucose feed started at 7 hours of elapsed fermentation time (EFT), after the glucose was close to $1 \mathrm{~g} \mathrm{I}^{-1}$. To determine concentrations of glucose, ethanol and the dry cell mass, we centrifuged the samples and collected the supernatant and biomass separately. Biomass samples were dried at $80^{\circ} \mathrm{C}$ for 48 hours. The cell concentration reached $3.26 \times 10^{8}$ cells ml $^{-1}$ in 24 hours, and cell viability remained above $96 \%$. The feed volume was $1,200 \mathrm{ml}$ in 17 hours of fed-batch growth phase, with the feeding rate ramping from 0 to $16 \mathrm{ml} \mathrm{l}^{-1} \mathrm{~h}^{-1}$. Although cells were in an aerated fermentation condition, a small quantity of ethanol $\left(20 \mathrm{~g} \mathrm{I}^{-1}\right)$ was produced before the beginning of anaerobicity.

\section{Anaerobic ethanol production phase}

After 24 hours, we exposed aerobic, glucose-limited cultures grown at a moderate specific growth rate to two simultaneous perturbations: a rapid depletion of oxygen and glucose feeding regulated by redox potential measurement. Nitrogen, instead of air, was used to sparge the fermentor. The gas flow rate was kept at $0.5 \mathrm{VVM}\left(2.5 \mathrm{I} \mathrm{min}^{-1}\right)$. The medium feed was controlled by BioCommand OPC software using online redox potential measurements as an on-line input. Redox potential

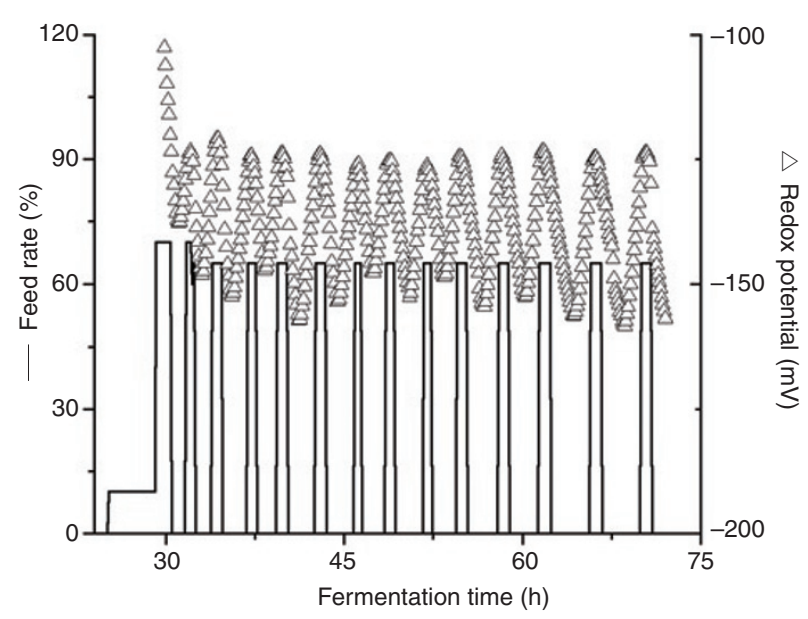

Figure 3 Use of redox potential to control the medium feed.

readings of $-130 \mathrm{mV}$ triggered a medium feed pump to add carbon source medium to maintain the oxidation-reduction level for the ethanol production (Fig. 3). Cell viability was monitored and measured, validating that cells remained healthy in the anaerobic production phase. Optical density values were maintained around 80 and viability at $88 \%$ at 72 hours elapsed fermentation time. Culture broth $\mathrm{pH}$ was well maintained at 5.0.

\section{Conclusion}

We cultured yeast in an aerobic fermentation and then switched to an anaerobic process to induce ethanol production, demonstrating how versatile the BioFlo 310 fermentor can be. We produced $85 \mathrm{~g} \mathrm{I}^{-1}$ of ethanol in 70 hours, while cell viability was maintained at levels as high as $88 \%$, proving this fermentor to be a very powerful and capable research or production instrument.

In addition to being used for a wide variety of fermentation processes, the BioFlo 310 can also be adapted for mammalian or insect culture with the use of optional accessories. Multiple connections are provided for integrating data from ancillary sensors, analyzers, scales or other devices for optimized process control. New Brunswick Scientific offers optional validation and training packages, as well as the services of an in-house lab to assist with process development and scale-up. For more information on this system or on our full range of advanced fermentors and bioreactors for research through production, see http://www.nbsc.com/n5c/ or write to us at bioinfo@nbsc.com.

1. Kwan, N.B., Hwang, T.S., Lee, S.H., Ahn, D.H. \& Park, D.H. Effect of electrochemica redox reaction on growth and metabolism of Saccharomyces cerevisiae as an environmental factor. J. Microbiol. Biotechnol. 17, 445-453 (2007).

This article was submitted to Nature Methods by a commercial organization and has not been peer reviewed. Nature Methods takes no responsibility for the accuracy or otherwise of the information provided. 